\title{
Un tuf volcanique semblable à l'Eltviller Tuff dans les loess de Hesbaye (Belgique) et du Limbourg néerlandais
}

\author{
Etienne Juvigné \& Arno Semmel *)
}

Volcanic tuff, Eltviller Tuff, correlation, loess, soil profile, hydromorphic soil, paleosol, gray forest soil, Upper Pleistocene, texture, C-14 dating, index map. Belgium (Hesbaye), Netherlands (Limburg), Upper Rhine Valley (Rheingau), Hessen, Rheinisches Schiefergebirge, Rheinland-Pfalz. TK 5408, 5914, 5915, 5916

R é s u mé : Un tuf volcanique a été mis en évidence dans trois profils de loess de Hesbaye (Rocourt et Lixhe) et du Limbourg néerlandais (Nagelbeek). Ce tuf correspond probablement à l'E $1 \mathrm{t}$ ville r T u f f connu en Hesse (Semmel 1967). En conséquence, cette découverte permet de comparer directement une partie de la séquence des loess du Pléistocène supérieur propre à chaque région.

\section{[Ein vulkanischer Tuff ähnlich dem Eltviller Tuff in den Lössen von Hesbaye (Belgien) und Niederländisch-Limburg]}

$\mathrm{Kurzf}$ assung: Ein vulkanischer Tuff ist in drei Lößprofilen von Ostbelgien (Rocourt und Lixhe) und niederländisch Limburg gefunden worden. Dieser Tuff kann mit dem E l t vill e r T u f f (Semmel 1967) korreliert werden. Daher können die Lößprofile von Ostbelgien, niederländisch Limburg (Nagelbeek) und Hessen parallelisiert werden.

\section{[A Volcanic Tuff Similar to the Eltviller Tuff in the Loess of Hesbaye (Belgium) and Limburg (Netherlands)]}

A bstract: A volcanic tuff has been found in three loess profiles of Hesbaye (Rocourt and Lixhe) and of Limburg in Netherland (Nagelbeek). This tuff corresponds probably with the Eltviller Tuff of Hessen in West Germany (SEmmeL, 1967). This key-bed permits to compare both loess stratigraphies.

\section{Introduction}

Rohdenburg \& Semmel (1971) ont décrit une très fine bande noire dans les loess de Rocourt (Belgique) sous un horizon rapporté par eux au sol de Kesselt (Fig. 1). Ils ont proposé une corrélation entre cette bande noire et un tuf volcanique connu en Hesse sous le nom d' E $1 \mathrm{t}$ vill e r Tuff (Semmel 1967). Sur base de leurs observations H. Rohdenburg et A. Semmel concluent à l'impossibilité d'une corrélation entre le sol de Kesselt et le sol de Stillfried B comme l'avait proposé Paepe (1966).

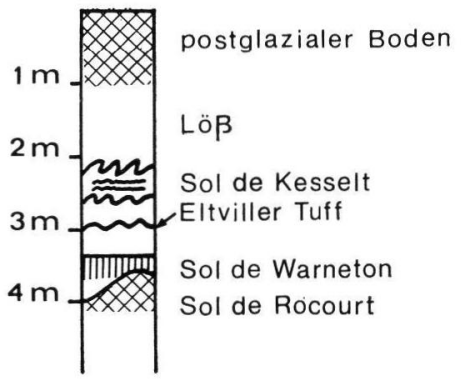

Fig. 1: Position de l'Eltville r Tuff à Rocourt, d'après Rohdenburg \& Semmel (1971, Abb. 1).

*) Anschrift der Verfasser: Dr. E. Ju vig n é, z. Zt. Forschungsstipendiat der Alexander-vonHumboldt-Stiftung, Universität zu Köln, Geologisches Institut, Lehrstuhl für Eiszeitenforschung, Zülpicher Straße 49, D-5000 Köln. - Chercheur qualifié au F.N.R.S., Université de Liège, Laboratoire de Géomorphologie et de Géologie du Quaternaire, Place du XX Août, 7, B 4000 Liège. Prof. Dr. A. S e m mel, Institut für Physische Geographie der Johann-Wolfgang-Goethe-Universität, Senckenberganlage 36, D-6000 Frankfurt am Main. 
Au cours de l'excursion organisée par la DEUQUA en septembre 1980, nous avons découvert dans les coupes de loess de Lixhe, et Nagelbeek ${ }^{1}$ ) une bande noire (Fig. 2) identique à celle signalée précédemment à Rocourt (RoHDenburg \& SEMmel 1971).

Dans le présent article, nous proposons une étude minéralogique et granulométrique de fines bandes noires de Hesbaye, du Limbourg néerlandais, de Hesse et du Palatinat. Les résultats plaident en faveur d'un tuf unique qui serait l'E $1 \mathrm{t}$ ville r Tuff.

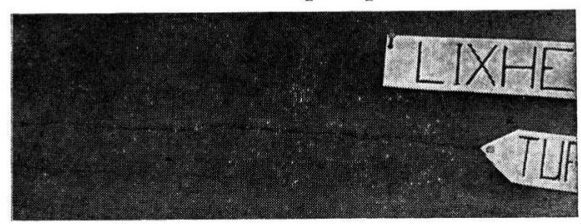

Fig. 2: Bande de tuf volcanique visible à l'oeil nu à Lixhe.

\section{ROCOURT}

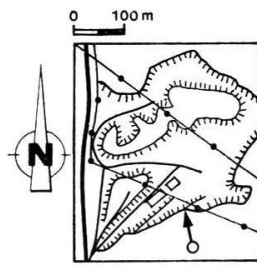

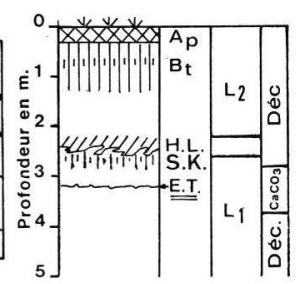

๑)

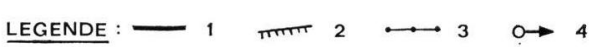

LIXHE

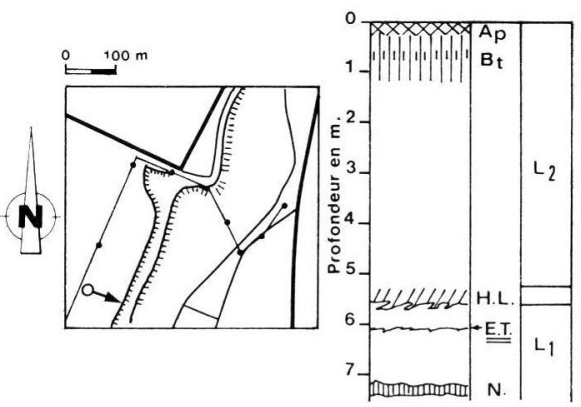

NAGELBEEK

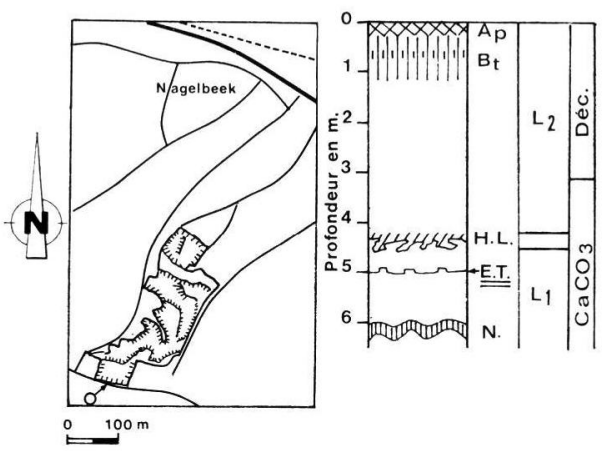

Fig. 3: Position du tuf volcanique dans trois profils de Hesbaye (Belgique) et du Limbourg néerlandais. Les plans de situation sont extraits de cartes topographiques au 10.000e; ils se rapportent respectivement aux carrières suivantes: Rocourt: Sablière de la S.A. Sables et Graviers. Lixhe: Carrière de craie de la C.B.R. Nagelbeek: Carrière Brull.

Légende: 1. Route. 2. Front de taille. 3. Ligne électrique à haute tension. 4. Point d'observation. Ap. Horizon labouré. Bt. Sol actuel naturel. H.L. Horizon à la ngues. S.K. S ol d e $\mathrm{K}$ esselt. E.T. Eltville r Tuff. N. Naßboden. Déc. Horizon décalcifié. $\mathrm{CaCO}_{3}$. Horizon contenant du calcaire. L1 Limon poudreux inférieur. L2 Limon poudreux supérieur.

1) Ces coupes ont été présentées respectivement par F. Gullentops (Lixhe) et O. S. Kuyl et H. Mücher (Nagelbeek). Chacun nous a aimablement autorisé à publier notre découverte. Nous les remercions très vivement. 


\section{Description du tuf volcanique de Hesbaye et du Limbourg néerlandais}

\subsection{Localisation du tuf dans les trois profils}

La localisation géographique des profils étudiés est rapportée sur la figure 4 et la position stratigraphique du tuf sur la figure 3 .

Dans chacune des trois coupes deux générations de loess poudreux (L1 et L2, fig. 3) peuvent être distinguées. Elles sont séparées par un horizon dont la base se présente sous forme de langues obliques ( $h$ or i z on à $\mathrm{l}$ a ng ues).

La mince bande de tuf volcanique est incluse au sein du dépôt loessique inférieur (L1). Celui-ci est généralement finement stratifié et montre localement des petites cryoturbations. L'excellente préservation des structures sédimentaires de ce loess atteste qu'aucune pédogenèse ne l'a affecté du moins au niveau du tuf. Il en résulte que la retombée des poussières volcaniques a dû se faire sous climat froid et sec.

Dans le profil de Rocourt, entre l'horizon à langues et le tuf volcanique il existe peut-être un sol désigné sous le nom de sol de Kesselt (Gullentops 1954: p. 149: "Une preuve formelle" - de la présence du sol de Kesselt à Rocourt - "n'en existe cependant pas parce qu'il manque un horizon calcareux entre la couche 6 et le profil actuel.“

\subsection{Composition des diverses bandes de tuf de Hesbaye, du Limbourg néerlandais ainsi que de l'Eltviller Tuff}

Nos observations concernent tous les points de prélèvements représentés sur la figure 4.

Tous les échantillons ont été prélevés essentiellement au niveau de chaque bande noire mais une contamination par le loess ne peut être évitée quand la couche est millimétrique.

Pour limiter au maximum les confusions lors des déterminations microscopiques, l'essentiel de la composante loessique a été éliminé par tamisage à $63 \mu \mathrm{m}$. En raison du fait que des grains ont pu traverser les mailles en diagonale, seuls ceux de plus de $80 \mu \mathrm{m}$ ont été considérés à l'analyse. Pour éviter la dissolution sélective de minéraux, seule l'eau oxygénée bouillante a été utilisée pour disperser les agglomérats. Les séparations entre fractions denses et légères ont été effectuées par centrifugation dans le bromoforme (Juvigne 1979).

En premier lieu, il faut insister sur la nature basaltique attestée par la couleur noire du tuf en place.

En ce qui concerne les minéraux denses transparents, les spectres sont représentés dans le tableau 1. Nous y avons ajouté des déterminations effectuées antérieurement par J. Frechen sur des échantillons de l'E l tville r Tuff prélevés à Weilbach et Hahnstätten. Ces résultats ont été publiés dans Bibus (1973).

La différence est très faible entre nos spectres et ceux décrits par J. FRECHEN. Il s'agit d'un léger accroissement des pyroxènes aux dépens de l'olivine. Ceci peut tenir à des différences propres à la méthode de traitement ou de détermination. Dans ce sens, les facteurs les plus déterminants sont souvent la classe granulométrique et/ou la méthode de séparation (décantation ou centrifugation). Mais en l'absence de tout renseignement méthodologique relatif aux spectres de J. FrECHEN, il ne nous est pas possible de proposer une explication fondée.

Dans tous les échantillons, les pyroxènes monocliniques sont semblables. rares.

Leur forme est généralement trapue, parfois prismatique. Les formes dentelées sont 
En lumière naturelle, ils sont souvent verts avec des nuances brunâtres ou grisâtres. Plusieurs grains sont à peine colorés et ne présentent guère qu'un discret reflet vert très pâle.

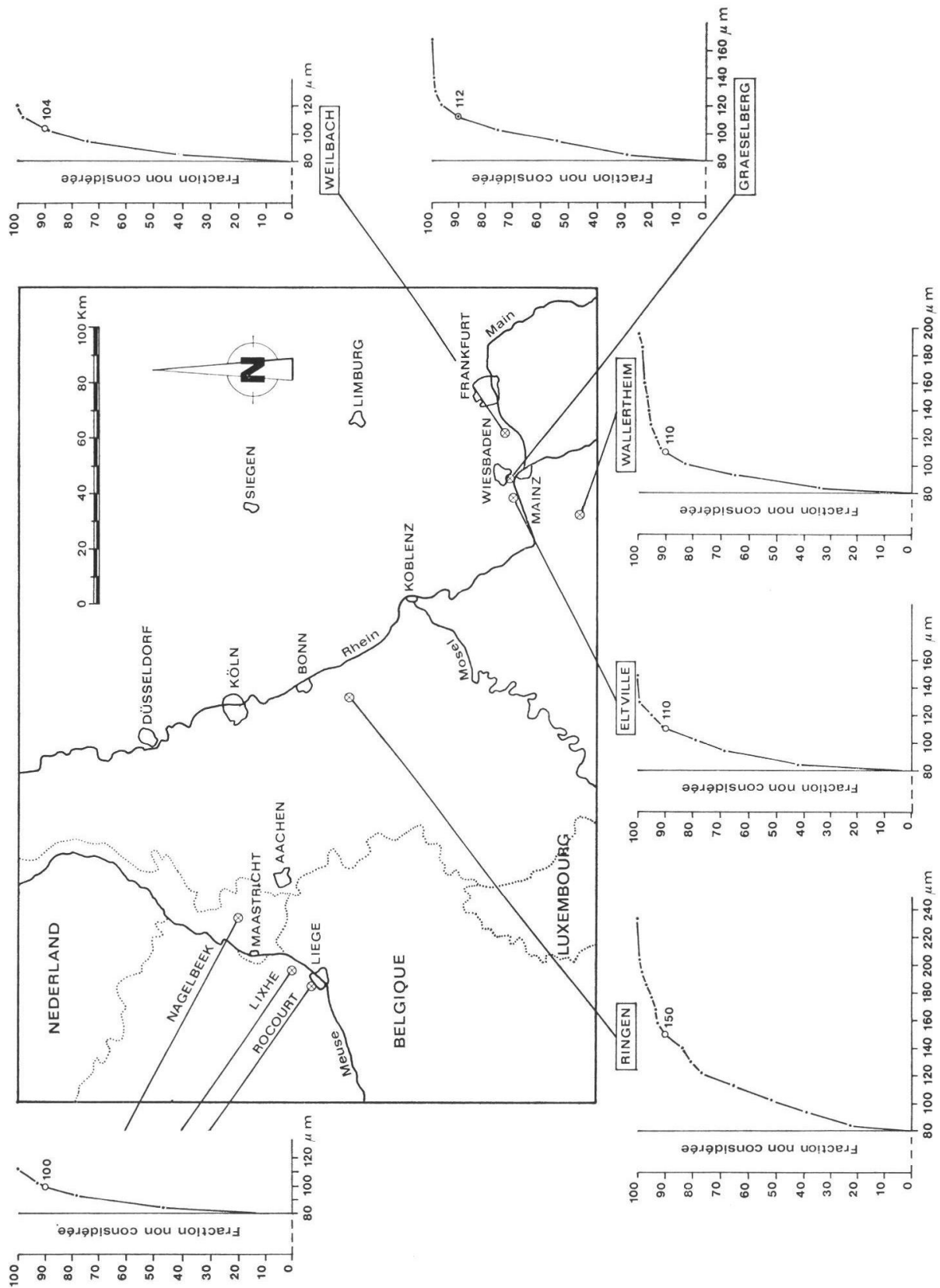

Fig. 4: Localisation des sites étudiés et granulométrie des pyroxènes monocliniques des diverses bandes de tufs volcaniques. 


\begin{tabular}{|c|c|c|c|c|c|c|c|c|c|c|}
\hline \multirow[b]{2}{*}{$\begin{array}{l}\text { Minéraux } \\
\text { denses } \\
\text { transparents }\end{array}$} & \multicolumn{2}{|c|}{ J. FRECHEN } & \multicolumn{8}{|c|}{ E. JUVIGNE } \\
\hline & 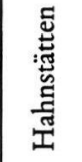 & $\begin{array}{l}\overline{\tilde{J}} \\
\hat{\bar{J}} \\
\overline{0}\end{array}$ & 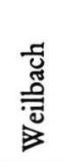 & 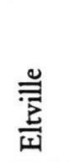 & 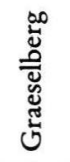 & 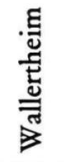 & 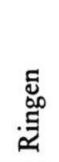 & 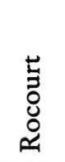 & 离 & 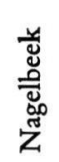 \\
\hline $\begin{array}{l}\text { Pyroxènes } \\
\text { monocliniques }\end{array}$ & 87,6 & 86,1 & 93,2 & 94,1 & 96,2 & 97,5 & 95,3 & 96,1 & 95,4 & 95,4 \\
\hline Olivine & 11,2 & 13,4 & 6,3 & 5,1 & 3,6 & 1,7 & 3,7 & 3,1 & 3,9 & 3,5 \\
\hline $\begin{array}{l}\text { Hornblende } \\
\text { brune }\end{array}$ & 1,2 & 0,5 & 0,4 & 0,8 & 0,3 & 0,9 & 1,0 & 0,9 & 0,7 & 1,1 \\
\hline
\end{tabular}

Tab. 1: Les associations de minéraux denses transparents de bandes de tufs volcaniques dans quelques sites de Hesse, du Palatinat, de Hesbaye et du Limbourg néerlandais.

En lumière polarisée, l'extinction est souvent incomplète, parfois même assortie d'une importante dispersion. Dans les tailles où les pyroxènes se présentent en Belgique et en Allemagne (fig. 4) il est rare que plus de deux ordres de teintes se manifestent.

\subsection{Granulométrie des pyroxènes monocliniques}

Nous rappelons d'abord que pour des raisons techniques $(\$ 2.2)$ seuls les grains de plus de $80 \mu \mathrm{m}$ ont été considérés. Dans chaque cas, c'est la plus grande largeur apparente au microscope qui a été mesurée à l'aide d'un oculaire gradué.

Les courbes propres à chaque site sont dessinées sur la figure 4. En raison des différences extrêmement faibles entre les sites belges et néerlandais, l'unique courbe rapportée vaut pour tous les profils.

Pour éviter les écarts exceptionnels qui peuvent se manifester quand on raisonne sur le plus gros grain, nous avons représenté sur chaque graphique la taille du grain correspondant au $90 \mathrm{e}$ pourcent de la fraction étudiée.

C'est à Ringen (Eifel) que les grains sont les plus gros; le 90e pourcent atteint $150 \mu \mathrm{m}$. C'est aussi dans ce site que le tuf est le plus épais. Il peut être sous-divisé en plusieurs fines couches ramifiées, distribuées sur une hauteur d'environ $5 \mathrm{~cm}$, mais l'épaisseur cumulée des fines bandes de tuf ne représente qu'environ $2 \mathrm{~cm}$. Ces caractéristiques nous indiquent que parmi les sites étudiés, Ringen est le plus proche du point d'émission qui serait donc nécessairement dans l'Eifel.

Le 90 e pourcent a une taille de $110 \mu \mathrm{m}$ à $112 \mu \mathrm{m}$ dans le triangle Eltville-Wallertheim-Graeselberg. A Weilbach, la taille du 90 e pourcent est un peu plus faible $(104 \mu \mathrm{m})$. C'est en Hesbaye et au Limbourg néerlandais que la bande de tuf est la plus fine (1 à $5 \mathrm{~mm})$ et que le $90^{\mathrm{e}}$ pourcent est le plus petit $(100 \mu \mathrm{m})$.

\subsection{Identité du tuf de Hesbaye et du Limbourg néerlandais}

Une corrélation a déja été proposée par Rohdenburg \& Semmel (1971) entre la bande noire de Rocourt et l'Eltviller Tuff. Ce rapprochement était seulement basé sur la position stratigraphique des tufs respectifs. Il nous parait utile d'insister sur le fait que la composition et la granulométrie plaident aussi en faveur d'une telle corrélation. 
Nous tenons aussi à rappeler ici que l'un d'entre nous (E. JUVIGNÉ) a antérieurement attribué à H. Rohdenburg et A. SEmmel une confusion de la bande noire de Rocourt avec le tuf de Rocourt (Juvigné 1977). Les présents résultats montrent que $H$. Rohdenburg et A. Semmel n'avaient pas effectué cette confusion.

\section{Le problème de l'âge du tuf}

Si comme nous, on admet que le tuf volcanique découvert dans les loess de Hesbaye et du Limbourg néerlandais correspond à l'Eltville r Tuff, alors on se trouve en présence de solutions contradictoires.

\subsection{Age de l'Eltviller Tuff d'après la stratigraphie de Hesse et du Palatinat}

L'Eltviller Tuff est défini comme une tuffitische Lage située entre les $\mathrm{Na} \beta$ böden $E_{3}$ et $E_{4}$ dans la stratigraphie des loess würmiens de Hesse méridionale représentée à la figure 5 (Semmel 1967). Dans cette stratigraphie, les $\mathrm{Na}$ B -

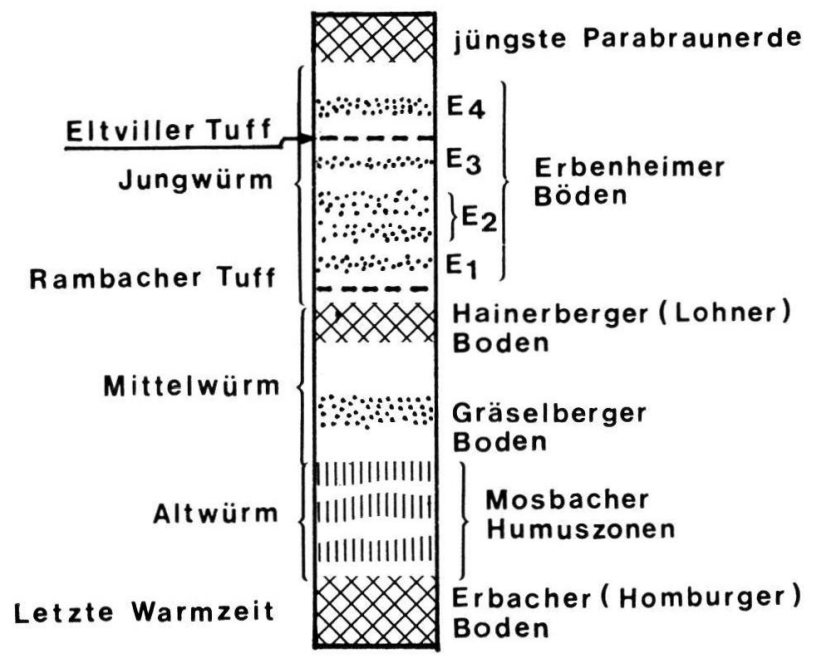

Fig. 5: Position de l'Eltville r Tuff dans la stratigraphie générale des limons de Hesse (d'après Semmel 1967, Abb. 1).

böden $E_{1}$ à $E_{4}$ divisent les loess du Würm récent à la base desquels se trouve le $\mathrm{H}$ a in erberger Boden ou Lohner Boden. Celui-ci est le sol le mieux développé dans les loess würmiens sus-jacents à l'horizon humifère du Würm ancien. Ceci vaut pour les loess d'Allemagne Fédérale mais aussi pour les loess du "F e u c h te L ö ß landschaft" d'Autriche (FINK 1976), où le Lohner Boden est visible par exemple dans la coupe de la briqueterie de Feilendorf au sud-ouest de Sint Poelten (Bibus \& Semmel 1977). J. Fink défend l'idée que ce sol est l'équivalent du sol de Stillfried B dans la "Trockenen Lößlandschaf t". Si l'on admet cette corrélation et l'âge $14 \mathrm{C}$ du sol de Stillfried B, alors l'Eltviller Tuff se trouve dans un loess qui est nécessairement plus jeune que 28000 ans.

Dans le cas où l'on contesterait ces considérations relatives à la valeur stratigraphique du Lohner Boden, il n'en reste pas moins que d'autres données confirment que l'E $1 \mathrm{t}$ ville r T u f ferait nécessairement plus jeune que 30000 ans.

1) Deux datations absolues ont été réalisées par le Laboratoire $14 \mathrm{C}$ du "Niedersächsisches Landesamt für Bodenforschung " sur des mollusques prélevés dans le $\mathrm{Na} ß$ b o d e n $\mathrm{E}_{2}$ sous- jacent au tuf. Elles ont donné respectivement: 
- Hv $1296: 18.500 \pm 950 \mathrm{BP}$

- Hv $1297: 21.100 \pm 1400 \mathrm{BP}$

Ces deux datations sont rapportées par Semmel 1967.

2) Un reste de crâne d'H o mo sa pie n s s a piens a été découvert dans les sédiments de la terrasse $t_{6}$ du Main sur laquelle existe localement des dépôts loessiques contenant l'E $1 \mathrm{tv}$ ille r Tuff. Une datation $14 \mathrm{C}$ de ce reste de crâne a fourni un âge de $31200 \pm 600$ B.P. En outre une détermination basée sur les acides aminés a été réalisée par R. Protsch; elle a donné un âge de 32000 B.P. (Protsch \& Semmel 1978).

3) Au lieu-dit Napoleonshöhe près de Sprendlingen (Palatinat), l'E $1 \mathrm{t} v \mathrm{i} l 1$ e r Tuff a été trouvé dans les loess surmontant une couche archéologique rapportée au Gravettien (Bosinski 1979). Ivanova (1972) a proposé une série de datations $14 \mathrm{C}$ pour des sites archéologiques du Gravettien; dans l'ensemble les résultats sont plus jeunes que 30000 B.P. Sans ignorer la problématique relative aux datations $14 \mathrm{C}$ on ne peut cependant admettre sur base des données actuelles un âge plus ancien que 30000 ans pour l'Eltviller Tuff. Sur base de l'âge des coquilles du $\mathrm{Na}$ ß bo de $\mathrm{n} \mathrm{E} \mathrm{E}_{2}$, un âge plus jeune que 20000 ans est même dans le domaine des probabilités.

\subsection{Age de l'Eltviller Tuff d'après la stratigraphie des loess de l'Eifel et de la Niederrheinische Bucht}

LÖHr et BrunNaCKer (1974) ont observé l'Eltviller Tuff dans une douzaine de profils de loess de l'Eifelvorland septentrional et de la Niederrheinische Bucht. Il se présente entre le I I I. B o d e n qui est mis en corrélation avec l'in te r s t a de de L a u ge rieLascaux (environ 18.000 B.P.) et le II. Boden parallélisé avec le sol de Stillfried B.

\subsection{Age du tuf en Hesbaye et au Limbourg néerlandais d'après la stratigraphie locale}

Le tuf volcanique se présente en dessous de l'horizon à 1 a ng u es et peut-être même sous le sol de Kesselt. Gullentops (1957) a proposé une corrélation entre le sol de Kesselt et l'interstade Würm II/Würm III (Bordes 1954). D'après ce dernier, cet interstade doit être situé entre le Paléolithique moyen et le Paléolithique supérieur.

Ultérieurement, à Kesselt, BASTIN (1971) a mis en évidence par la palynologie une amélioration climatique au sein de l'h orizon à $\mathrm{l}$ a ngues. Il a désigné cette phase d'amélioration climatique sous le nom d'oscillation de Kesselt et l'a située au sein de l'interstade d'Arcy-Stillfried B pour lequel il propose un âge compris entre 32.500 B.P. et 28.500 B.P.

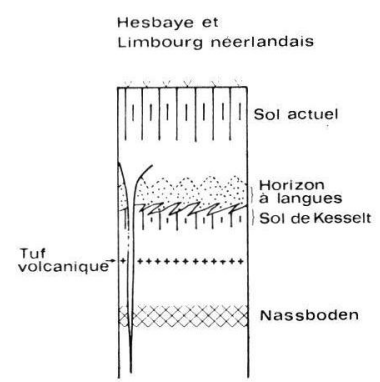

Fig. 6: Position du tuf volcanique dans la stratigraphie générale des limons de Hesbaye et du Limbourg néerlandais. (Rappel: la position du Sol de Kesselt dans cette Figure n'est pas certaine: revoir $\mathrm{p} 85$.) 


\section{Conclusion}

Si l'on considère comme exacte la chronostratigraphie des limons de Hesse ainsi que celle des limons de Belgique et du Limbourg néerlandais, alors il existerait deux tufs volcaniques de même composition minéralogique mais d'âge nettement distinct.

Par contre, si l'on admet comme nous l'existence d'un même tuf dans chacune des deux régions en cause, alors il y a lieu de revoir les chronostratigraphies respectives.

\section{Bibliographie}

BAStin, B. (1971): Recherches sur l'évolution du peuplement végetal en Belgique durant la glaciation de Würm. - Acta Geographica Lovaniensia, 9: 136 p.; Louvain.

Bibus, E. (1973): Ausbildung und Lagerungsverhältnisse quartärer Tuffvorkommen in der Wetterau. - Notizbl. hess. L.-Amt Bodenforsch., 101: 346-361; Wiesbaden.

- \& Semmel, A. (1977): Stratigraphische Leithorizonte im Würmlöß des Mittelrheingebietes. Geol. Jb. Hessen, 105: 141-147; Wiesbaden.

Bordes, F. (1954): Les limons quaternaires du Bassin de la Seine. Stratigraphie et Archéologie paléolithique. - Arch Inst. Paléont. Hum., 26: 472 p.; Paris.

Bosınsкт, G. (1979): Ein Fundplatz des mittleren Jungpaläolithikums bei Sprendlingen, Kreis Mainz-Bingen. - Archäologisches Korrespondenzblatt, 9: 147-153; Mainz.

Fink, J. (1976): Ziegelwerk Feilendorf in: Exkursion durch den österreichischen Teil des nördlichen Alpenvorlandes und den Donauraum zwischen Krems und Wiener Pforter. - Mitt. Komm. Quartärforsch. österr. Ak. Wiss., 1: 62-64; Wien.

Gullentops, F. (1954): Contributions à la chronologie du pléistocène et des formes du relief en Belgique. - Mém. Inst. Géol. Univ. Louvain, 18:123-252; Louvain.

- (1980): Lixhe - Grube C.B.R. Führer zur Exkursion 2 der Deutschen Quartärvereinigung vom 14. bis 16. 9. 80, S. 56-58.

Ivanova, I. K. (1972): Das geologische Alter des fossilen Menschen. - Archaeologica Venatoria, 1: 224 S.; Stuttgart.

Juvigne, E. (1977): Zone de dispersion et âge des poussières volcaniques du tuf de Rocourt. Ann. Soc. Géol. Belg., 100: 13-22; Liège.

- (1979): Scheidetrichtermethode oder Zentrifugaltrennung zur quantitativen Gewinnung von Schwermineralien. - Senckenbergiana Marit., 11: 171-174; Frankfurt a. M.

KUYL, O. S. \& MÜCHER, H. (1980): Nagelbeek-Kiesgrube Brull: Lößstratigraphie NiederländischSüdlimburgs. - Führer zur Exkursion 2 der Deutschen Quartärvereinigung vom 14. bis 16. 9. 80 , S. $84-95$.

LöHr, H. \& Brunnacker, K. (1974): Metternicher und Eltviller Tuff-Horizont im Würm-Löß am Mittel- und Niederrhein. - Notizbl. hess. L.-Amt Bodenf., 102: 168-190, Wiesbaden.

PAEPE, R. (1966): Comparative stratigraphy of Würm loess deposits in Belgium and Austria. Bull. Soc. Belge de Géol., 75: 203-216; Bruxelles.

Рвотsch, R. \& Semmel, A. (1978): Zur Chronologie des Kelsterbach-Hominiden des ältesten Vertreters des Homo sapiens sapiens in Europa. - Eiszeitalter u. Gegenwart, 28: 200-210; Ohringen.

Rohdenburg, H. \& Semmel, A. (1971): Bemerkungen zur Stratigraphie des Würm-Lösses im westlichen Mitteleuropa. - Notizbl. hess. L.-Amt Bodenforsch., 99: 246-252; Wiesbaden.

Semmel, A. (1967): Neue Fundstellen von vulkanischem Material in hessischen Lössen. - Notizbl. hess. L.-Amt Bodenforsch., 95: 104-108; Wiesbaden.

P.S. En cours de publication E. MeIJs nous a fait savoir qu'il déposait dans la revue Geologie en Mijnbouw une courte note relative à la découverte de l'Eltviller Tuff dans les environs de Maastricht (Pays-Bas). La parution est prévue en 1981.

Manuskript eingegangen am 10. 12. 1980.

Remerciements: Au cours de la préparation de ce travail, le Dr. P. Haesaerts, le Dr. B. Bastin et le Professeur A. Pissart ont bien voulu discuter avec nous du problème posé par la découverte du tuf volcanique dans les loess de Hesbaye et du Limbourg néerlandais. Ils ont ainsi largement contribué à la valorisation du manuscript. Nous les en remercions très vivement. 